This is the author's copy of the publication as archived with the DLR's electronic library at http://elib.dlr.de . Please consult the original publication for citation, see e.g. https://ieeexplore.ieee.org/document/9177599

\title{
A New Fuzzy Logic Based Adaptive Motion Cueing Algorithm Using Parallel Simulation- Based Motion Platform
}

Mohammad Reza Chalak Qazani, Houshyar Asadi, Tobias Bellmann, Siamak Perdrammehr, Shady Mohamed, Saeid Nahavandi

Parallel manipulators are recently used in most motion simulation laboratories as they can easily generate six degrees of freedom motion. Recently, fuzzy logic-based adaptive motion cueing algorithms (MCAs) have been employed to reproduce the motion signals. The usage of fuzzy logicbased adaptive MCA reduces the movement sensation error between the real vehicle and the simulation-based motion platform (SBMP) user considering the end-effector limitations in Cartesian space.. In this paper, a new fuzzy logic-based adaptive MCA is introduced to generate motion signals based on the joints' limitations and the movement sensation error between the real vehicle and the SBMP user. Considering the parallel SBMP, joint limits enhance the ability of the introduced adaptive motion cueing algorithm to generate more accurate movement feelings with high fidelity. The simulation results prove that the proposed adaptive motion cueing algorithm can effectively use the large workspace of the parallel SBMP whilst reducing the motion sensation error.

\section{Copyright Notice}

(C2020 IEEE. Personal use of this material is permitted. Permission from IEEE must be obtained for all other uses, in any current or future media, including reprinting/republishing this material for advertising or promotional purposes, creating new collective works, for resale or redistribution to servers or lists, or reuse of any copyrighted component of this work in other works.

M. R. Chalak Qazani, H. Asadi, T. Bellmann, S. Perdrammehr, S. Mohamed and S. Nahavandi, "A New Fuzzy Logic Based Adaptive Motion Cueing Algorithm Using Parallel Simulation-Based Motion Platform," 2020 IEEE International Conference on Fuzzy Systems (FUZZ-IEEE), Glasgow, United Kingdom, 2020, pp. 1-8, doi: 10.1109/FUZZ48607.2020.9177599. 


\section{A New Fuzzy Logic Based Adaptive Motion Cueing Algorithm Using Parallel Simulation-Based Motion Platform}

\author{
Mohammad Reza Chalak Qazani \\ the Institute for Intelligent Systems \\ Research and Innovation \\ Deakin University \\ Geelong, VIC 3125, Australia \\ m.r.chalakqazani@gmail.com
}

\author{
Houshyar Asadi \\ the Institute for Intelligent Systems \\ Research and Innovation \\ Deakin University \\ Geelong, VIC 3125, Australia \\ houshyar.asadi@deakin.edu.au
}

\author{
Tobias Bellmann \\ Institute of System Dynamics and \\ Control, Space Systems Dynamics, \\ Deutsches Zentrum für Luft- und \\ Raumfahrt (DLR) \\ German Aerospace Center \\ 82234, Germany \\ Tobias.Bellmann@dlr.de
}

Saeid Nahavandi

the Institute for Intelligent Systems

Research and Innovation

Deakin University

Geelong, VIC 3125, Australia

saeid.nahavandi@deakin.edu.au

\author{
Siamak Perdrammehr \\ the Institute for Intelligent Systems \\ Research and Innovation \\ Deakin University \\ Geelong, VIC 3125, Australia \\ s.pedrammehr@gmail.com
}

\author{
Shady Mohamed \\ Research and Innovation \\ Deakin University \\ Geelong, VIC 3125, Australia \\ shady.mohamed@deakin.edu.au
}

\begin{abstract}
Parallel manipulators are recently used in most motion simulation laboratories as they can easily generate six degrees of freedom motion. Recently, fuzzy logic-based adaptive motion cueing algorithms (MCAs) have been employed to reproduce the motion signals. The usage of fuzzy logic-based adaptive MCA reduces the movement sensation error between the real vehicle and the simulation-based motion platform (SBMP) user considering the end-effector limitations in Cartesian space. . In this paper, a new fuzzy logic-based adaptive MCA is introduced to generate motion signals based on the joints' limitations and the movement sensation error between the real vehicle and the SBMP user. Considering the parallel SBMP, joint limits enhance the ability of the introduced adaptive motion cueing algorithm to generate more accurate movement feelings with high fidelity. The simulation results prove that the proposed adaptive motion cueing algorithm can effectively use the large workspace of the parallel SBMP whilst reducing the motion sensation error.
\end{abstract}

Keywords- motion cueing algorithm, adaptive controller, fuzzy logic, motion platform, path planning

\section{INTRODUCTION}

The application of simulation-based motion platforms increases due to their tremendous beneficial points in many areas such as transportation system, human psychology, reliability to autonomous, car factory and aerospace industry [1-3]. They can be used as a handy device to train pilots or even test newly designed cars even before rapid prototyping them. Most motion simulation laboratories use parallel manipulators as an SBMP because it is easy to reach high acceleration using some low-load actuators [4-6]. Parallel SBMPs are very cost-effective, easy to control and provide high stiffness and acceleration [7-9]. Moreover, there are analytical solutions to extract the inverse kinematic model of parallel SBMPs. However, the parallel SBMPs have a highly restricted workspace regarding linear and angular motions. Recently automotive companies, such as Benz or Renault [10], have attached their parallel SBMPs to linear guides in order to increase its linear workspace area. The usage of linear guides eliminates the high linear workspace restriction of the parallel SBMP along $x$ - and $y$-axis.

The movement signals of the real vehicle cannot be directly implemented into the SBMP because of the limited workspace area. The approach to reproduce the real vehicle motion signals (including linear acceleration and angular velocity), generating the same real vehicle movement feeling for the SBMP user, is called motion cueing algorithm (MCA) including classical [11, 12], adaptive [13-18], optimal [19-23] and model predictive control [24-28]. The classical MCAs are very popular in industries and research centres because of its simplicity, easy tunning, low computational load and safety. Classical MCAs filter the low-frequency part of the motion signal using a highpass filter to drive the SBMP inside the workspace area. In addition, classical MCAs reproduce sustainable linear accelerations using a low-pass filter combined with somatogravic illusion [29]. Qazani et al. [12] monitored the performance index of the hexapod SBMP using classical MCA. Classical MCA does not consider the SBMP limitation in generating the motion signal, and then it should be tuned considering the worst-case scenario to respect the SBMP limitation in every motion scenario. However, the worst-case tuning technique reduce the efficiency by generating false motion cues. The damping ratio and fixed cut-off frequencies of the classical MCA, as well as the lack of consideration for 
the human vestibular model, are other drawbacks of classical MCAs. Parrish et al. [18] introduced the first adaptive MCA. The adaptive MCA for 2-DoF SBMPs using the gradient descent optimization method, to extract the optimal coefficient parameters of the filters based on the motion sensation error and the position and velocity limitations of the SBMP, was developed by Nehaoua et al. [17]. Pradipta and Sawodny [15] introduced the actuator constrained MCA for a pneumatic hexapod platform. They employed the online optimisation method to find the best motion signals based on the current position of the SBMP and the actuators' limitations. Since their method uses the online optimisation algorithm, there is a high computational load for real-time applications. Ellensohn et al. [14] used the direct kinematics model of the SBMP and the prediction model of the motion signal to extract the best endeffector configuration based on the position, velocity and acceleration limitations of the actuators. Their model suffered from high computational loads since the direct inverse kinematics problem cannot be solved analytically, and the prediction model increases the size of the matrices that should be solved in real-time. The fuzzy logic controller is employed to generate the motion signal taking into account the Cartesian workspace limitations and human motion sensation errors by Asadi et al. [13, 16]. Considering the workspace limits of SBMP in the Cartesian system instead of the joints' limitations can reduce the efficiency of the method because of the conservative use of the workspace area.

The CAD model and the closed kinematic chain of the Stewart simulation platform can be seen in Fig. 1. The limitations of the parallel SBMP end-effectors and its corresponding joint space limits along the longitudinal channel have also been shown in Table I.

All the previous studies on adaptive MCAs [13-18] considered the limitations of the SBMP in the Cartesian system instead of the joints' limitations which lead to the conservative use of the workspace area. Considering the active and passive joints' positions in adaptive MCA leads to the extensive use of the mechanisms workspace and improves the fidelity for the driver of the SBMP. The main contribution of this study is to propose an adaptive fuzzy logic-based MCA to generate a correction signal based on the SBMPs active and passive joints' limits and the human sensation error between the real vehicle and driver of the SBMP. The online consideration of the joints' limits of the parallel SBMP eliminates the conservative use of the workspace that leads to simulation artefacts and motion sickness. As a result, the better usage of the workspace leads to better generation of the high-pass and low-pass motion signal with high fidelity for the SBMP user.

The inverse kinematics model of the parallel SBMP is explained in Section II. The fuzzy logic-based adaptive MCA is illustrated in Section III. Moreover, the simulation is discussed in Section IV. The concluding comments are discussed in Section V.

\section{InVERSE Kinematics Model of The Parallel SBMP}

Inverse kinematics is the mathematical process of calculating the joint parameters needed to position the SBMP legs with a desired velocity and acceleration considering the

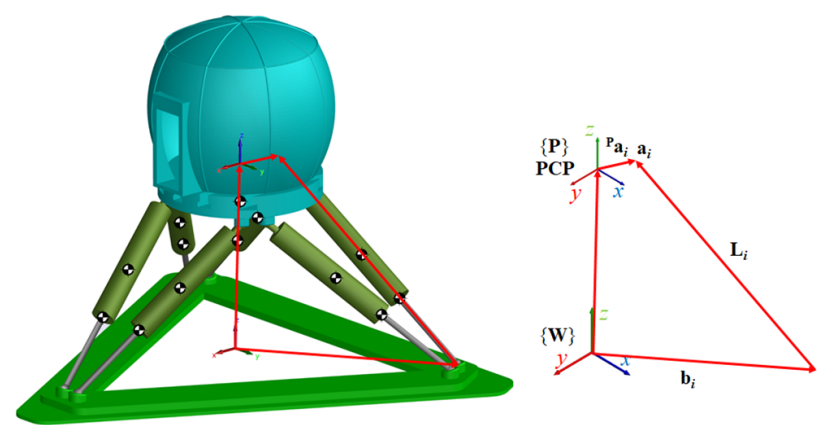

Fig. 1. (a): The CAD model of the parallel SBMP; (b): The kinematic chain of the parallel SBMP.

current position, velocity, and acceleration of the platform. Fig. 1 illustrates the kinematic chain of the Stewart simulation platform manipulator.

The $i^{\text {th }}$ leg's length vector kinematic chain, $\mathbf{L}_{i}$, can be obtained as:

$\mathbf{L}_{i}+\mathbf{b}_{i}=\mathbf{P C P}+\mathbf{a}_{i}$

in which $\mathbf{a}_{i}$ is the $i^{\text {th }}$ joint's position vector on the upper platform, and $\mathbf{b}_{i}$ is the $i^{\text {th }}$ joint's position vector on the lower platform.

Considering PCP as the platform position vector in the base coordinate frame, $\mathbf{a}_{i}$ can be obtained as:

$\mathbf{a}_{i}=\mathbf{R}^{\mathrm{P}} \mathbf{a}_{\mathrm{i}}$

in which ${ }^{\mathrm{P}} \mathbf{a}_{\mathrm{i}}$ is the $i^{\text {th }}$ upper joint's position vector with respect to frame $\{\mathbf{P}\}$.

The rotation matrix along XYZ, which relates the rotation of $\{\mathbf{P}\}$ to $\{\mathbf{W}\}$ has been shown by $\mathbf{R}$. the rotation matrix can be obtained as:

$\mathbf{R}=\left[\begin{array}{ccc}\mathrm{c} \phi \mathrm{c} \theta & \mathrm{c} \phi \mathrm{s} \theta \mathrm{s} \psi-\mathrm{s} \phi \mathrm{c} \psi & \mathrm{c} \phi \mathrm{s} \theta \mathrm{c} \psi+\mathrm{s} \phi \mathrm{s} \psi \\ \mathrm{s} \phi \mathrm{c} \theta & \mathrm{s} \phi \mathrm{s} \theta s \psi+\mathrm{c} \phi \mathrm{c} \psi & \mathrm{s} \phi \mathrm{s} \theta \mathrm{c} \psi-\mathrm{c} \phi \mathrm{s} \psi \\ -\mathrm{s} \theta & \mathrm{c} \theta s \psi & \mathrm{c} \theta \mathrm{c} \psi\end{array}\right]$,

in which $\phi, \theta$, and $\psi$ respectively represent the roll, pitch, and yaw angles of the platform along $x, y$ and $z$ directions. Also, c and $s$ are the abbreviations of $\cos$ and $\sin$ functions respectively.

$l_{i i}$ is also the $i^{\text {th }}$ leg's length and can be obtained as:

$\mathbf{L}_{i}=l_{i} \mathbf{n}_{i}$

Considering Eq. (4), Eq. (1) can be rewritten as:

$l_{i} \mathbf{n}_{i}=\mathbf{P C P}+\mathbf{R}^{\mathrm{P}} \mathbf{a}_{\mathrm{i}}-\mathbf{b}_{i}$

There are two different types of passive joints in the structure of the Stewart simulation platform. The universal passive joints connect the fixed platform (base) to the legs, and the spherical passive joints attach the legs to the moving platform of the Stewart simulation platform. The spherical joints typically have higher angular limitations compared to the universal joints. Therefore, the passive upper joints should be extracted to be considered inside the adaptive MCA. The angular displacement of the $i^{\text {th }}$ upper passive joints, $\beta_{i}$, can be found by taking the dot product between the normal vector of the legs' position and the normal vector of the Stewart simulation platform's position as follows:

$\beta_{i}=\frac{\mathbf{n}_{i} \cdot \mathbf{n}_{P C P, i}}{\left|\mathbf{n}_{i}\right|\left|\mathbf{n}_{P C P, i}\right|}$ 
TABLE I

THE WORKSPACE BOUNDARIES OF THE PARALLEL SBMP IN LONGITUDINAL CHANNEL

\begin{tabular}{cccc}
\hline \hline Index & Position & Velocity & Acceleration \\
\hline$l_{i}$ & $0.5-1.4(\mathrm{~m})$ & $\pm 1.5(\mathrm{~m} / \mathrm{s})$ & $\pm 2\left(\mathrm{~m} / \mathrm{s}^{2}\right)$ \\
$\beta_{i}$ & $\pm 17(\mathrm{deg})$ & NA & NA \\
X & $\pm 0.45(\mathrm{~m})$ & $\pm 1.3(\mathrm{~m} / \mathrm{s})$ & $\pm 3.3\left(\mathrm{~m} / \mathrm{s}^{2}\right)$ \\
Pitch & $\pm 10(\mathrm{deg})$ & $\pm 61(\mathrm{deg} / \mathrm{s})$ & $\pm 600\left(\mathrm{deg} / \mathrm{s}^{2}\right)$ \\
\hline \hline
\end{tabular}

m: meter; s: second; deg: degree.

where $\mathbf{n}_{P C P, i}$ are the spherical shell positions of the $i^{\text {th }}$ joints situated at the end-effector.

Considering the legs' positions, velocities and accelerations, as well as the angular displacements of the passive upper joints, can enhance the ability of the proposed algorithm to reproduce the more accurate movement signals based on the position, velocity, and acceleration of the Stewart simulation platform. The whole workspace area of the Stewart simulation platform cannot be used efficiently due to the conservative assumption of the workspace limitations in the Cartesian dimension.

\section{FuZZY LOGIC-BASED ADAPTIVE MCA}

Considering the joints' limits, the schematic structure of the classical and proposed adaptive MCAs are shown in Fig. 2. The driver's action based on its vision generates the motion signal including linear acceleration $\left(a_{x}\right)_{\text {veh }}$ and angular velocity $(\dot{\theta})_{v e h}$ signals which are the inputs of the MCA.

Fig. 2a illustrates the structure of the classical MCA, which consists of a second-order low-pass and high-pass filters in the translational and tilt coordination channels. Moreover, a firstorder high-pass filter is employed in the rotational channel as:

$$
\begin{aligned}
& H P_{\text {Trans }}=\frac{s^{2}}{s^{2}+2 \xi \omega_{n} s+\omega_{n}^{2}} \\
& L P_{\text {Trans }}=\frac{\omega_{n}^{2}}{\mathrm{~s}^{2}+2 \xi \omega_{n} s+\omega_{n}{ }^{2}} \\
& H P_{\text {Rot }}=\frac{\mathrm{s}}{\mathrm{s}+\omega_{n}}
\end{aligned}
$$

where $\omega_{n}$ and $\xi$ are respectively the cut-off frequency and damping ratio of the mentioned filters. The classical MCA does not consider the physical limits of SBMP, and it should be tuned based on the worst-case scenario to keep the driver within the workspace. It also ignores the human vestibular model when regenerating the motion signals.

The human vestibular model is located inside the inner ear of the human body as the main part of the human perceptual system, which is responsible for sensing the motions. This system is composed of the otolith organ and semicircular canals to sense the translational and rotational motion signals.

The best transfer function models to represent the human vestibular system are [30-32]:

$\frac{\hat{f}}{f}=K_{\text {OTO }}\left(\frac{\left(\tau_{a} s+1\right)}{\left(\tau_{L} s+1\right)\left(\tau_{s} s+1\right)}\right)$
$\frac{\widehat{\omega}}{\omega}=\left(\frac{\tau_{1} \tau_{b}{ }^{2}}{\left(\tau_{b} s+1\right)\left(\tau_{1} s+1\right)}\right)$

where $\hat{f}$ and $\widehat{\omega}$ are respectively the sensed specific force and

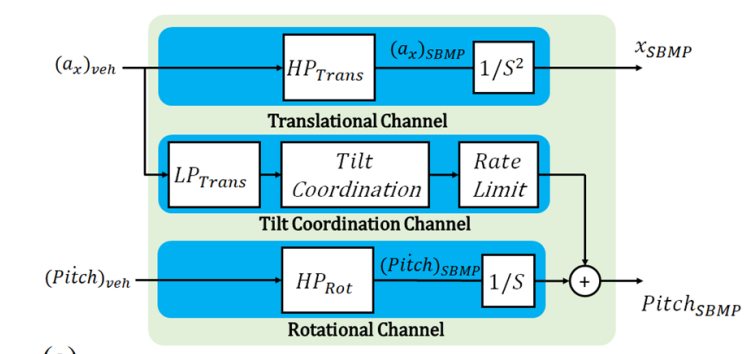

(a)

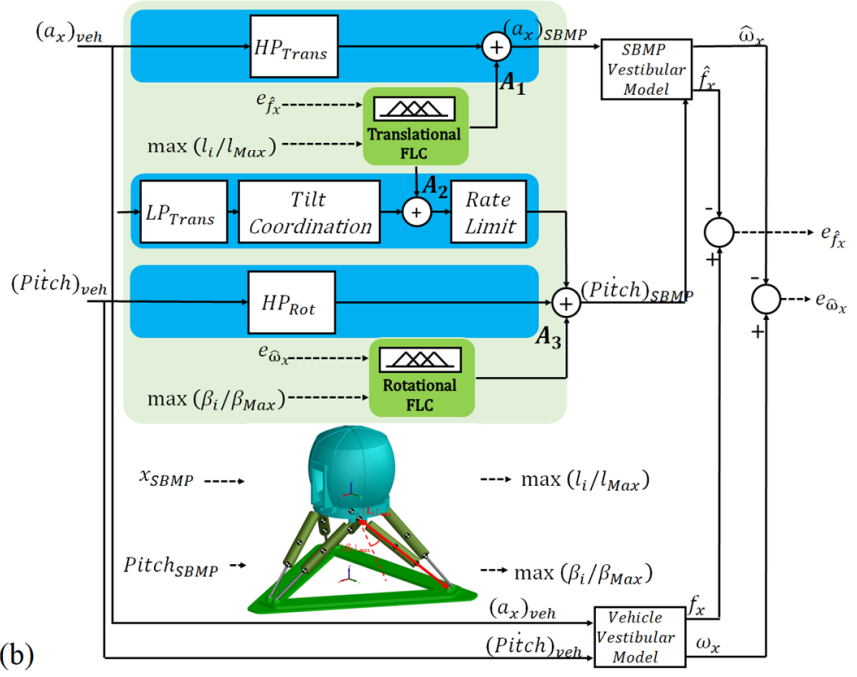

Fig. 2. The schematic structure of MCAs for longitudinal channel (a): the classical MCA; (b): the proposed fuzzy logic-based adaptive MCA.

sensed angular velocity, $f$ and $\omega$ are also the applied specific force and applied angular velocity, $K_{\text {ото }}=0.4, \tau_{a}=$ $13.2(s), \tau_{s}=0.016(s), \tau_{a}=13.2(s), \tau_{a}=30(s)$ and $\tau_{1}=5.3(s)$ in the longitudinal channel.

Fig. $2 \mathrm{~b}$ presents the structure of the proposed adaptive MCA using the fuzzy logic controller to generate the compensation motion signal in three channels: rotational, translational and tilt coordination channels. There are two fuzzy logic units in the longitudinal channel. The inputs of the fuzzy logic units are the motion sensation error between the real-world vehicle driver and SBMP driver as well as the maximum displacement of the joints divided into its angular and linear displacement limits. The inverse kinematic model extracts the linear positions of active joints and angular positions of the passive joints and splits them into their corresponding maximum linear and angular displacements. The highest displaced joint should be implemented in the fuzzy unit as it is the critical joints to reach the limitations between six joints. The vestibular model also calculates the error of the motion feeling online based on the generated motion via the MCA. According to Fig. 2b, the fuzzy units generates three fuzzy control signals including A1, A2, and A3 based on the motion feeling error considering the user of the SBMP and the maximum linear and angular displacement of the active and passive joints, respectively. The fuzzy control signals A1 and A2 are respectively implemented into the translational and tilt coordination channels. They have 
been employed to generate more accurate high- and low-pass sustainable acceleration motion signals, based on the current situation of SBMP, reducing the specific force error sensed by the SBMP driver. The fuzzy control signal A3 is added to the rotational channel of the MCA to reduce the sensed angular velocity error.

\section{A. Fuzzy Logic Control Unit}

The structure of the fuzzy logic controller for the translational and rotational channels of the longitudinal direction of the MCA has been shown in Fig. 3. The inputs of the translational fuzzy logic unit are the specific force error sensed by the SBMP driver and the maximum displacement of the joints. The first input should be normalised with the otolith threshold, which is $0.17\left(\mathrm{~m} / \mathrm{s}^{2}\right)$ along the longitudinal channel, before inputting it into the fuzzy unit. The second input of the fuzzy translational unit has already been normalised as it has been divided by the angular displacement limits of the joints. The fuzzy scale block is also employed to increase the efficiency of the fuzzy logic controller, and it should be tuned based on the joints' limitation of the parallel SBMP. To separate the high- and low-pass frequency parts of the motion signal, there is a high-pass filter unit in the translational fuzzy logic channel. The high-pass frequency part of the generated fuzzy control $\left(A_{1}\right)$ is added to the translational channel of the MCA to use the linear workspace area of the parallel SBMP more efficiently. The low-pass frequency part of the fuzzy control signal is also divided by gravity and added to the channel of tilt coordination. This reduces the error of the sensed specific force. Fig. 3b represents the structure of the rotational fuzzy block controller. The rotational fuzzy controller inputs are the error of sensed angular velocity by the user of the SBMP and the maximum displacement of the joints, same as the second input of the translational fuzzy logic controller. The second input has already been normalised; however, the first input should be normalised by diving by the semicircular threshold which is $3(\mathrm{deg} / \mathrm{s})$ in this study. The generated fuzzy control signal is scaled based on the workspace limitation of the parallel SBMP and filtered using a high-pass filter to keep the angular displacement of the driver inside the limitations.

\section{B. Membership Function and Rule}

It is not possible to sense the quantity of the motion sensation via the human vestibular system. The fuzzy logic controller, on the other hand, can be employed to process the features of the human vestibular system model. Indeed, the vestibular system is capable of sensing the motion sensation in various channels including longitudinal, lateral, heave and yaw. As a result, the motion sensation error for the angular velocity and linear acceleration signals between the real driver and the SBMP driver can be categorised in five groups which are large negative, negative, zero, positive, and large positive. The angular positions of the joints can be extracted using the inverse kinematics model in Section II. They are divided into

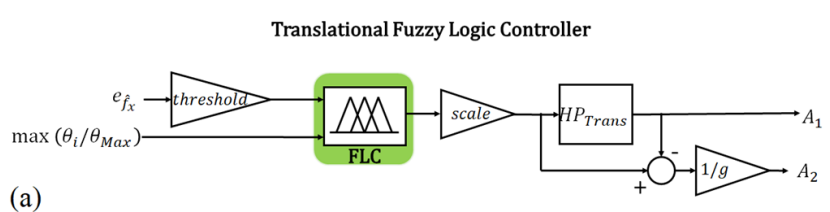

(a)

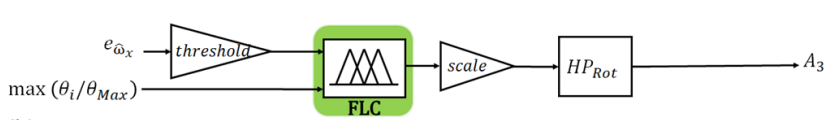

(b)

Fig. 3. The structure of the proposed adaptive MCA (a): translational fuzzy logic controller; (b): rotational fuzzy logic controller.

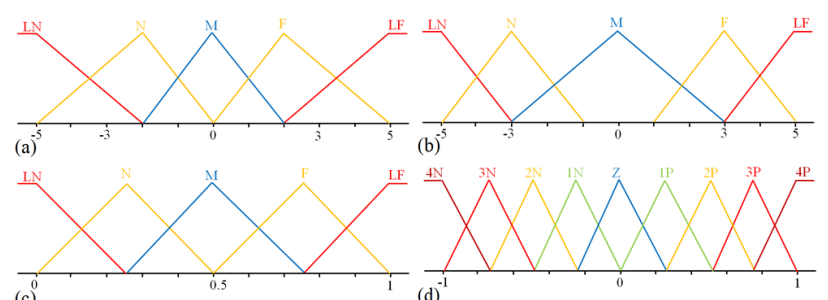

Fig. 4. (a): Membership function of the linear motion sensation error; (b): Membership function of the angular motion sensation error; (c): Membership function of the maximum position limitations of joints; (d): Membership function of the fuzzy output control.

the angular limitations, and the maximum extracted value is employed as the second input of the fuzzy units in translational and rotational channels. It has been split into five different ranges as large near, near, medium, far and large far.

Fig. 4 shows the membership functions of the fuzzy logic controller for the fuzzy logic-based adaptive MCA using joints' limitations. Figs. 5a and 5b illustrate the membership functions of the fuzzy control output, translation motion sensation error, and rotational motion sensation error, respectively.

There is a big difference between the input membership functions of the rotational and translational motion sensation error (that is the dead zone in the structure of the rotational sensation error membership function). It has been defined to modify the error of the sensed angular velocity on the human semicircular threshold, while the translational motion sensation error membership function is developed to reduce the translational motion sensation error, continuously. Fig. 4c shows the membership function of the second input of the fuzzy logic controller known as the maximum displacement of the parallel SBMP's actuators and passive joints. Fig. 4d represents the membership function of the fuzzy control output which is divided into nine groups known as 4-times negative, 3-times negative, 2-times negative, 1-times negative, zero, 1-times positive, 2-times positive, 3-times positive and 4-times positive. These membership functions are designed based on the trial-and-error and the knowledge of the system to reach the goal, which is the reduction of motion sensation error between the real and SBMP drivers. Among the trapezoidal, triangular, sigmoid, and Gaussian membership function, the triangular membership function is chosen in this study because of the light computational burden, loading a 
TABLE II

FUZZY CONTROL RULES FOR LINEAR DISPLACEMENT WEIGHT PARAMETER TUNING

\begin{tabular}{ccccccc}
\hline \hline \multirow{2}{*}{$W_{x}$} & & & $e_{\hat{\mathrm{f}}_{x}}$ & & \\
& & $\mathrm{LN}$ & $\mathrm{N}$ & $\mathrm{Z}$ & $\mathrm{P}$ & $\mathrm{LP}$ \\
\hline $\max \left(\theta_{i}\right)$ & $\mathrm{LN}$ & $4 \mathrm{~N}$ & $3 \mathrm{~N}$ & $\mathrm{Z}$ & $3 \mathrm{P}$ & $4 \mathrm{P}$ \\
$\operatorname{limitation}\left\{\theta_{i}\right\}$ & $\mathrm{N}$ & $3 \mathrm{~N}$ & $2 \mathrm{~N}$ & $\mathrm{Z}$ & $2 \mathrm{P}$ & $3 \mathrm{P}$ \\
& $\mathrm{F}$ & $2 \mathrm{~N}$ & $1 \mathrm{~N}$ & $\mathrm{Z}$ & $1 \mathrm{P}$ & $2 \mathrm{P}$ \\
& $\mathrm{LF}$ & $\mathrm{Z}$ & $\mathrm{Z}$ & $\mathrm{Z}$ & $\mathrm{Z}$ & $1 \mathrm{P}$ \\
\hline \hline
\end{tabular}

LN: large negative and large near; N: negative and near; Z: zero; P: positive; LP: large positive; M: middle and medium; F: far; LF: large far; $1 \mathrm{~N}$ : 1-times negative; 2N: 2times-negative; 3N: 3-times negative; 4N: 4-times negative; 1P: 1-times positive; 2P: 2-times positive; 3P: 3-times positive; 4P: 4-times positive.

small amount of data, simple definition using the asymmetric form.

The rules of the fuzzy logic controller are shown in Table II, which are the Mamdani type fuzzy. According to Table II, the translational human sensation error and the joints angular position are inputs. The control outputs generate the right selection of the fuzzy rules. For instance, if the error of the sensed specific force between the SBMP and real driver is very positive, and the maximum angular position of the joints is close to limits, the fuzzy unit will not generate the correction motion signal with respect to the joints limits. The sensed angular velocity error or sensed specific force error change the output when joints are close to the neutral position.

\section{RESUlt AND Discussion}

The classical MCA [33], previous adaptive MCA with consideration of the parallel SBMP workspace limitation in the Cartesian system introduced by Asadi et al. $[13,16]$ and the newly proposed adaptive MCA in Section III are modelled using MATLAB software. Also, the parallel SBMP is developed using SimMechanics environment of MATLAB software to consider the dynamical and physical limitations of the joints. The SimMechanic model increases the capability of the model by producing more realistic results. It should be mentioned that the same scale factor is employed for three MCAs (1/5 and $1 / 2$ for translational and rotational motion signal, respectively) to reach a fair evaluation of them [34, 35].

The simulation platform known as Rigs of Rods (RoR) has been employed to generate the angular velocity and linear acceleration motion signals for 140 (s) as shown in Fig. 5 which is the comprehensive motion scenario including sudden acceleration, turning the steering wheel, deceleration and braking.

Fig 6.a presents the rotational motion sensation for the user of the real vehicle and SBMP users using the classical, previous adaptive and proposed adaptive MCAs during 40 to 65 seconds motion scenario. The correlation coefficient can be employed to compare the shape similarity of two signal, which is introduced by Asadi et al. [1]. The CC varies between -1 and 1 , and the higher value of CC means more similarity between the shapes of the two signals. The shape similarity of

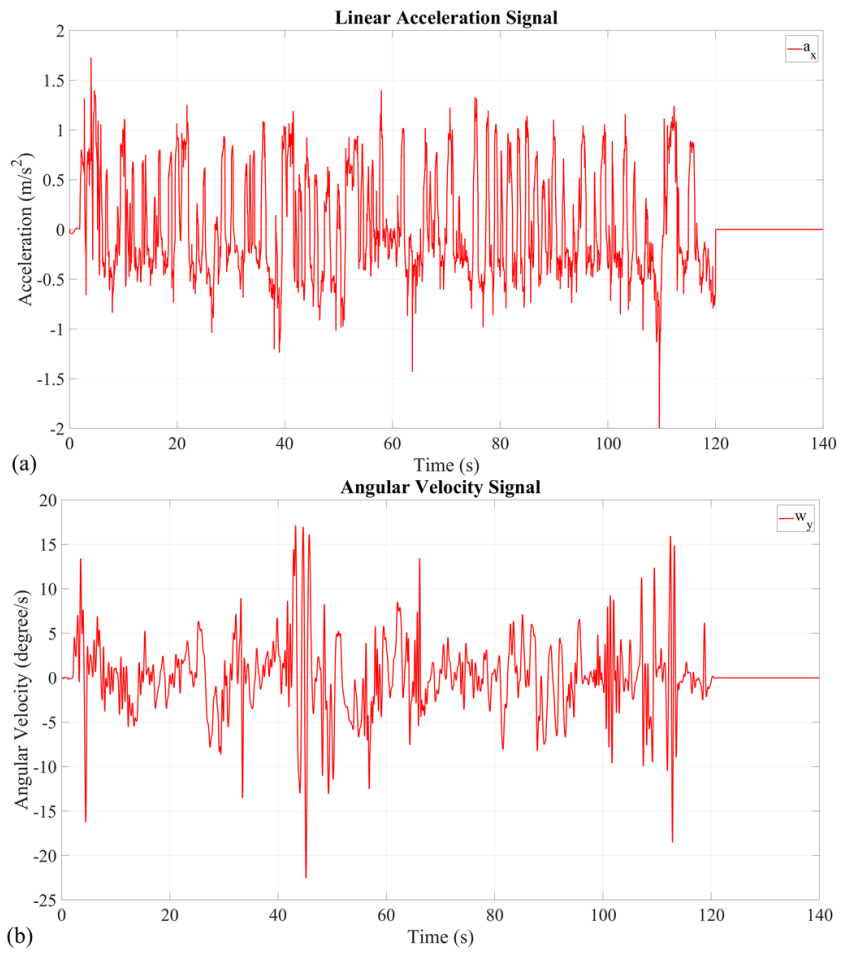

Fig. 5. The reference signal (real vehicle motions): (a) linear acceleration; (b) angular velocity.
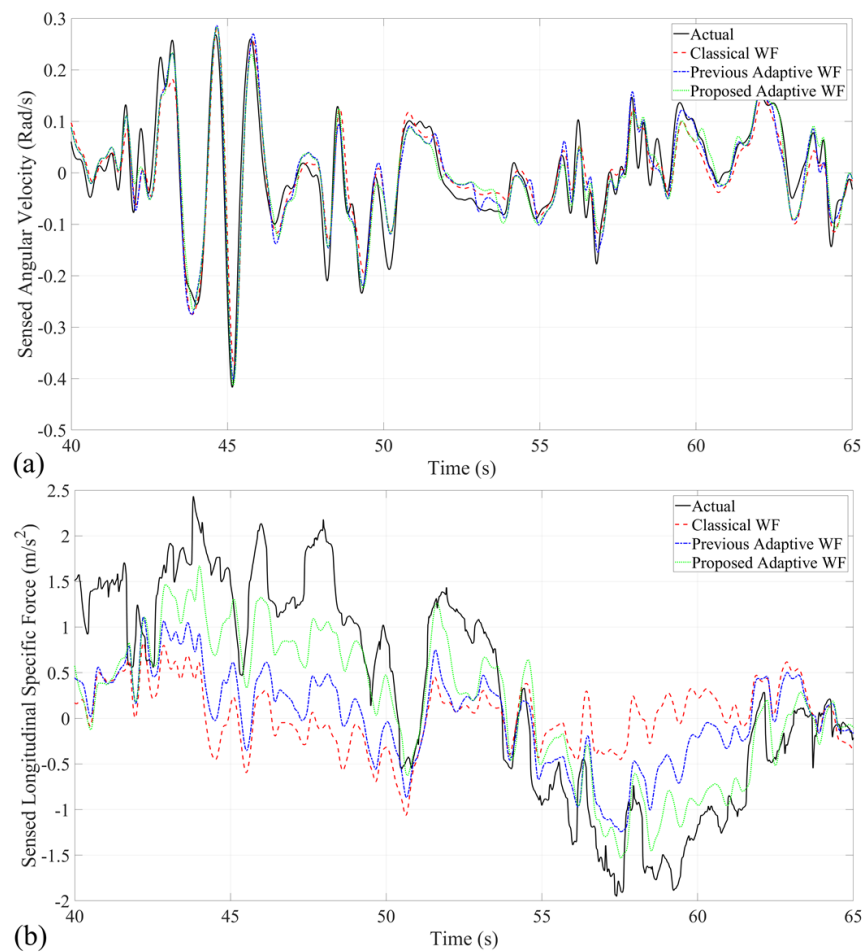

(b)

Fig. 6. (a): The rotational motion sensation; (b): The translational motion sensation, for the driver of the real vehicle and SBMP using the classical, pervious adaptive and proposed MCAs along $\mathrm{x}$-axis.

the rotational motion sensation using proposed adaptive MCA enhances 0.68 and 0.1 percent compared with classical and pervious adaptive MCAs because of the generated motion compensation signal based on the real-time consideration of 
TABLE III

The Performance IndeXes of the Proposed Adaptive, Previous ADAPTIVE AND CLASSICAL MCAS

\begin{tabular}{c|ccc}
\hline \hline \multirow{2}{*}{\multicolumn{2}{|l}{ Index }} & $\begin{array}{c}\text { SSF along } \\
x \text {-axis }\end{array}$ & $\begin{array}{c}\text { SAV along } \\
\text { pitch-angle }\end{array}$ \\
\hline \multirow{2}{*}{$\begin{array}{c}\text { Root Mean } \\
\text { Square Error }\end{array}$} & Classical MCA & 0.6602 & 0.0303 \\
\cline { 2 - 4 } & Previous Adaptive MCA & 0.4929 & 0.0299 \\
\cline { 2 - 4 } & Proposed Adaptive MCA & 0.3649 & 0.0296 \\
\hline \multirow{2}{*}{$\begin{array}{c}\text { Correlation } \\
\text { Coefficient }\end{array}$} & Classical MCA & 0.3497 & 0.8937 \\
\cline { 2 - 4 } & Previous Adaptive MCA & 0.7703 & 0.9003 \\
\cline { 2 - 4 } & Proposed Adaptive MCA & 0.8725 & 0.9013 \\
\hline \hline
\end{tabular}

SSF: Sensed specific force along $x$-axis; SAV: Sensed angular velocity

the motion sensation error and joints limitations including active and passive joints of hexapod SBMP. Fig. 6.b also represents the translational motion sensation for the real vehicle driver and the user of the parallel SBMP obtained by classical, previous adaptive and newly proposed adaptive MCAs during 40 to 65 seconds motion scenario. The better shape similarity of the translational motion sensation using the new proposed adaptive MCA is evident as the CC of the newly proposed adaptive MCA improves from 0.3947 and 0.7703 to 0.8725 better than classical and previous adaptive MCA along the x-axis, respectively. The proposed fuzzy logic-based adaptive MCA can consider the limits of the joints and use the workspace area wisely compared to the classical and previous adaptive MCAs. The insufficient usage of the workspace leads to the generation of false motion cues, which are the main reason for motion artefacts and motion sickness of the SBMP driver. The shape similarity factors of the rotational and translational motion sensation using three investigated methods are presented in the third column of table III.

Traditionally, root means square error (RMSE) is employed to validate the MCAs. Lower RMSE means the highly efficient motion cues for the SBMP user compared with real driver. The human semicircular canals cannot sense the angular velocity under the human rotational motion sensation threshold unit. The maximum sensed angular velocity error between the real vehicle and SBMP drivers is of importance to tune the investigated methods not to violate the human rotational motion sensation threshold unit. Fig. 7.a illustrates the rotational motion sensation error between the user of the real vehicle and SBMP using classical, previous adaptive and proposed adaptive MCAs along pitch-angle during 40 to 65 seconds motion scenario. It should be noted that the rotational motion sensation error using three investigated methods are under the human rotational motion sensation threshold unit using three methods. Also, the RMSE of the rotational motion sensation using proposed adaptive MCA decreases 2.31 and 1.00 percent compared with classical and previous adaptive MCAs. In addition, Fig. 7.b presents the motion sensation error along $x$-axis for the user of SBMP compared with real vehicle using three methods during 40 to 65 seconds motion scenario. The RMSE of the longitudinal motion sensation is $0.6602,0.4929$ and $0.3649(\mathrm{~m} / \mathrm{s} 2)$ using classical, previous adaptive and proposed adaptive MCAs. Then, the proposed
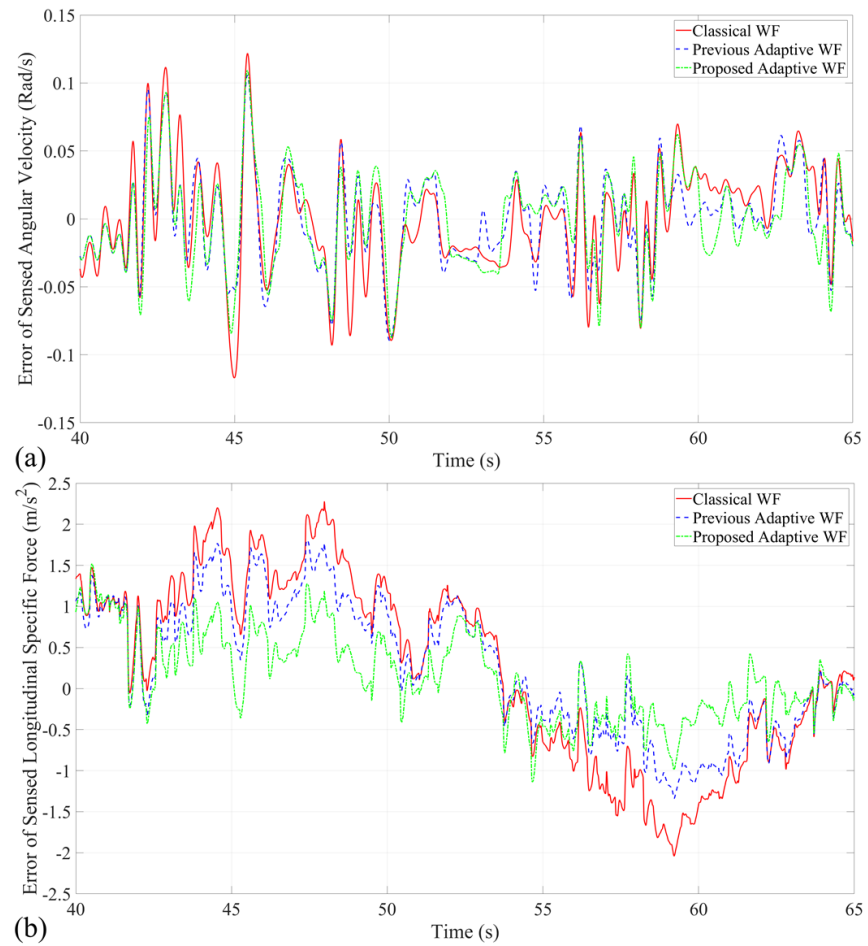

(b)

Fig. 7. (a): The rotational motion sensation error; (b): The translational motion sensation error, between the driver of the real vehicle and SBMP using the classical, pervious adaptive and proposed MCAs along $x$-axis.

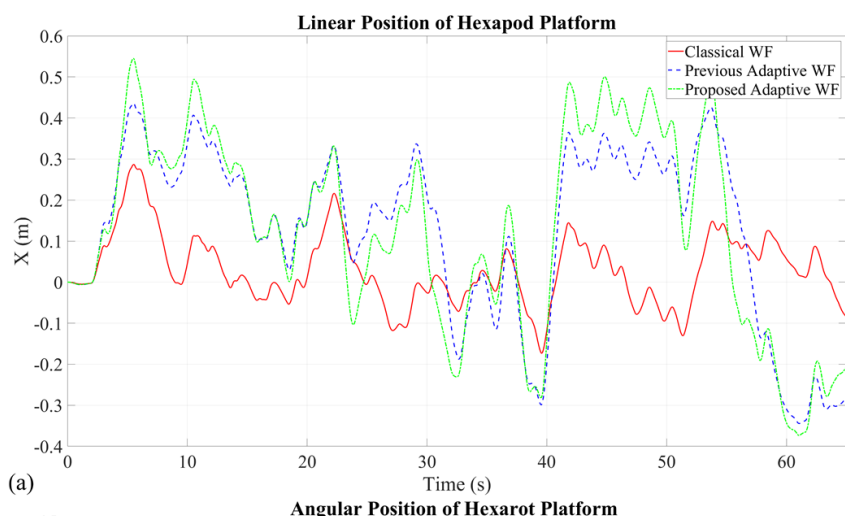

(a)

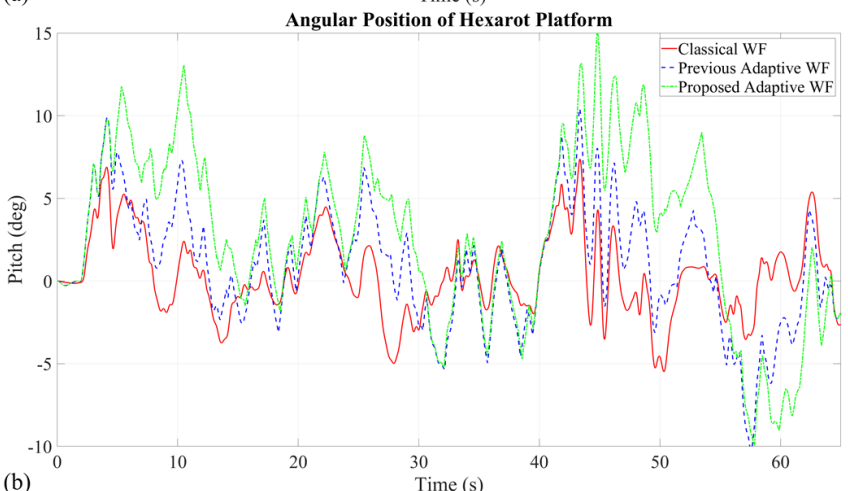

Fig. 8. (a): Linear displacement of the parallel SBMP using classical, previous adaptive and proposed adaptive MCAs along $x$-axis; (b): Angular displacement of the parallel SBMP using classical, previous adaptive and proposed adaptive MCAs along pitch-angle.

adaptive MCA decreases the longitudinal motion sensation error 44.72 and 25.97 percent compared with classical and 

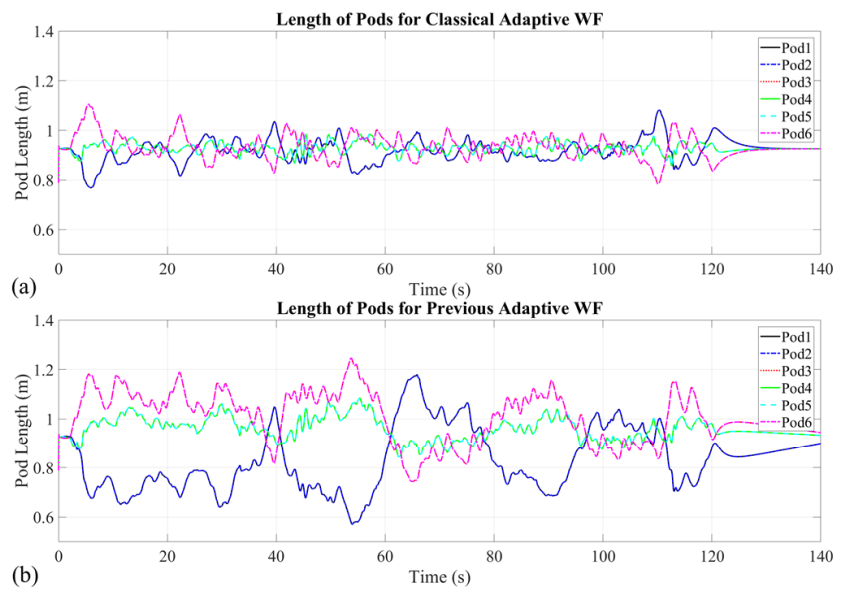

(b)

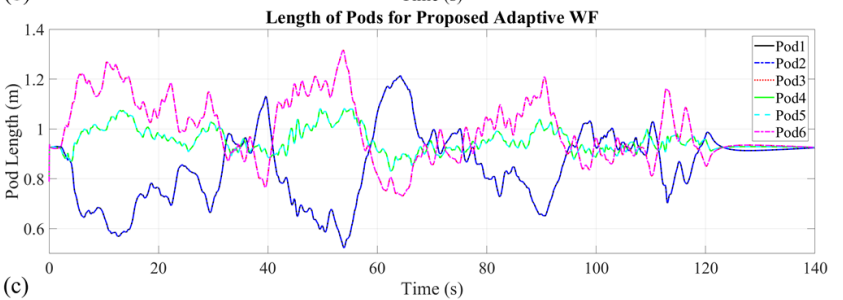

(c)

Fig. 9. Pods' length of the parallel SBMP (a): of the classical MCA; (b): of the previous adaptive MCA; (c) of the proposed adaptive MCA.

pervious adaptive MCAs. It should be noted that the improvement of the translational motion sensation is important because it does not cause the reduction in the rotational motion sensation. Table III shows the performance indexes of the classical, previous adaptive and proposed adaptive MCAs to prove the advantages of the proposed method with consideration of the inverse kinematic problem.

The angular and linear displacements of the driver obtained by classical, pervious adaptive and proposed adaptive MCAs are respectively shown in Fig. 8.a-b, respectively for duration of 65 seconds motion scenario. The Proposed adaptive MCA consider the actuators and passive joints' limitations, then it can use the workspace area more wisely compared with classical and previous adaptive MCAs. The proposed fuzzy logic-based adaptive MCA can use the rotational motion of the cockpit better than the classical and previous adaptive MCAs to produce more accurate sustainable low-frequency linear acceleration signal. Also, the rotational fuzzy logic controller is able to consider the rotational motion sensation error, respecting with it the human rotational motion sensation threshold, generating accurate rotational motion signals. Fig. 9 shows the legs length obtained by classical, pervious adaptive and proposed adaptive MCAs. Fig. 9c presents that the proposed adaptive MCA uses the actuators position limitations better than the classical and previous adaptive MCAs as they do not consider the joints' limits. Therefore, the classical and previous adaptive MCAs should be tuned conservatively.

The obtained results prove that the fuzzy logic-based adaptive MCA is efficient enough compared with classical and previous adaptive MCA. It can be used with for different mechanisms, if their inverse kinematic model is adequately developed. The better regeneration of the high-pass and lowpass frequency motion signals is the key to the improvement using the proposed method.

\section{CONCLUSION}

In this study, a fuzzy logic-based adaptive MCA was proposed to consider the actuators and passive joints limits of the Stewart platform parallel-based SBMP to generate the intact driving movement sensation. The classical MCA cannot consider the joint limits of the SBMP in a Cartesian workspace, and it should be tuned according to the worst-case scenario to respect the workspace limits of the parallel SBMP. Moreover, the existing adaptive MCAs consider the physical limits of SBMP in the Cartesian system of the end-effector, while the physical limits of the joints are the main reason for the workspace limitation of the parallel SBMP. Considering the parallel SBMP limitations in the Cartesian system leads to the reduced usage of the workspace area, and it causes the regeneration of the wrong motion cues and artefacts. The objective of this paper was to better use of the parallel SBMP workspace area without any conservative usage. The inverse kinematics model of the parallel SBMP was studied to be considered in the proposed adaptive MCA. In addition, the fuzzy logic controller was employed to generate the compensation motion signal in translational and rotational channels. The fuzzy logic unit monitors the physical limitations of the joints as well as the motion sensation error and regenerates the suitable motion signal based on the current situation of the parallel SBMP. Triangular membership functions were employed because of their fast response and simplicity. The model was designed using MATLAB software and compared with classical and previous adaptive MCAs. The better usage of the workspace with respect to the joints' limitations was recorded using the proposed fuzzy logic-based adaptive MCA and compared to the classical and previous adaptive MCAs. In future studies, the dynamical behaviour of the SBMP will be considered by developing a new adaptive MCA to generate more accurate motion cues based on the dynamical limitation of the mechanism, such as actuators. In the future study, the dynamical behaviour of the SBMP will be considered in developing the new adaptive MCA to generate the more accurate motion cues based on the dynamical limitation of the mechanism such as actuators. Also, neural networks and deep learning [36-41] can be employed to in a new generation of the adaptive MCAs.

\section{REFERENCES}

[1] A. Seefried et al., "Multi-domain flight simulation with the DLR robotic motion simulator," in 2019 Spring Simulation Conference (SpringSim), 2019, pp. 1-12: IEEE.

[2] H. Asadi, S. Mohamed, C. P. Lim, and S. Nahavandi, "Robust optimal motion cueing algorithm based on the linear quadratic regulator method and a genetic algorithm," IEEE Transactions on Systems, Man, and Cybernetics: Systems, vol. 47, no. 2, pp. 238-254, 2016.

[3] T. Bellmann, J. Heindl, M. Hellerer, R. Kuchar, K. Sharma, and G. Hirzinger, "The dlr robot motion simulator part i: Design and setup," in 
2011 IEEE International Conference on Robotics and Automation, 2011,pp. 4694-4701: IEEE.

[4] M. R. C. Qazani, H. Asadi, and S. Nahavandi, "A New Gantry-TauBased Mechanism Using Spherical Wrist and Model Predictive Control-Based Motion Cueing Algorithm," Robotica, pp. 1-22, 2019.

[5] S. Pedrammehr, M. R. C. Qazani, H. Asadi, and S. Nahavandi, "Control system development of a hexarotbased high-G centrifugal simulator," in The 20th IEEE International Conference on Industrial Technology IEEE-ICIT 2019, 2019.

[6] M. R. C. Qazani, H. Asadi, and S. Nahavandi, "High-Fidelity Hexarot Simulation-Based Motion Platform Using Fuzzy Incremental Controller and Model Predictive Control-Based Motion Cueing Algorithm," IEEE Systems Journal, 2019.

[7] M. R. C. Qazani, S. Pedrammehr, H. Abdi, and S. Nahavandi, "Performance Evaluation and Calibration of Gantry-Tau Paralle Mechanism," Iranian Journal of Science and Technology, Transactions of Mechanical Engineering, pp. 1-15, 2019.

[8] J.-P. Merlet, Parallel robots. Springer Science \& Business Media, 2006.

[9] S. Pedrammehr, M. R. C. Qazani, H. Asadi, and S. Nahavandi, "Kinematic manipulability analysis of hexarot simulators," in The 20th IEEE International Conference on Industrial Technology, IEEE-ICIT 2019, 2019, pp. 13-15.

[10] F. Colombet, M. Dagdelen, G. Reymond, C. Pere, F. Merienne, and A. Kemeny, "Motion cueing: What is the impact on the driver's behavior," in Proceedings of the Driving Simulation Conference, 2008, pp. 171181.

[11] S. F. Schmidt and B. Conrad, Motion Drive Signals for Pilot Flight Simulators. National Aeronautics and Space Administration, 1970.

[12] M. R. C. Qazani, H. Asadi, S. Pedrammehr, and S. Nahavandi, "Performance analysis and dexterity monitoring of hexapod-based simulator," in 2018 4th International Conference on Control, Automation and Robotics (ICCAR), 2018, pp. 226-231: IEEE.

[13] H. Asadi, C. P. Lim, S. Mohamed, D. Nahavandi, and S. Nahavandi, "Increasing Motion Fidelity in Driving Simulators Using a FuzzyBased Washout Filter," IEEE Transactions on Intelligent Vehicles, vol. 4, no. 2, pp. 298-308, 2019.

[14] F. Ellensohn, F. Oberleitner, M. Schwienbacher, J. Venrooij, and D. Rixen, "Actuator-Based Optimization Motion Cueing Algorithm," in 2018 IEEE/ASME International Conference on Advanced Intelligent Mechatronics (AIM), 2018, pp. 1021-1026: IEEE.

[15] J. Pradipta and O. Sawodny, "Actuator constrained motion cueing algorithm for a redundantly actuated stewart platform," Journal of Dynamic Systems, Measurement, and Control, vol. 138, no. 6, p. 061007, 2016.

[16] H. Asadi, S. Mohamed, and S. Nahavandi, "Incorporating human perception with the motion washout filter using fuzzy logic control," IEEE/ASME Transactions on Mechatronics, vol. 20, no. 6, pp. 3276$3284,2015$.

[17] L. Nehaoua, H. Mohellebi, A. Amouri, H. Arioui, S. Espié, and A. Kheddar, "Design and control of a small-clearance driving simulator," IEEE Transactions on Vehicular Technology, vol. 57, no. 2, pp. 736746, 2008.

[18] R. V. Parrish, J. E. Dieudonne, and D. J. Martin Jr, "Coordinated adaptive washout for motion simulators," Journal of aircraft, vol. 12 , no. 1 , pp. 44-50, 1975.

[19] H. Asadi, S. Mohamed, D. Rahim Zadeh, and S. Nahavandi, "Optimisation of nonlinear motion cueing algorithm based on genetic algorithm," Vehicle System Dynamics, vol. 53, no. 4, pp. 526-545, 2015 .

[20] H. Asadi, S. Mohamed, K. Nelson, S. Nahavandi, and D. R. Zadeh, "Human perception-based washout filtering using genetic algorithm," in International Conference on Neural Information Processing, 2015, pp. 401-411: Springer.

[21] H. Asadi, S. Mohamed, K. Nelson, S. Nahavandi, and M. Oladazimi, "An optimal washout filter based on genetic algorithm compensators for improving simulator driver perception," in DSC 2015: Proceedings of the Driving Simulation Conference \& Exhibition, 2015, pp. 1-10: Max Planck Institute for the Advancement of Science.

[22] T. Bellmann, M. Otter, J. Heindl, and G. Hirzinger, "Real-time path planning for an interactive and industrial robot-based motion simulator," in Proc. of the 2nd Motion Simulator Conference, 2007.
[23] T. Bellmann, M. Otter, and G. Hirzinger, "The DLR robot motion simulator part II: Optimization based path-planning," in 2011 IEEE International Conference on Robotics and Automation, 2011, pp. 47024709: IEEE.

[24] A. Mohammadi, H. Asadi, S. Mohamed, K. Nelson, and S. Nahavandi, "Multiobjective and interactive genetic algorithms for weight tuning of a model predictive control-based motion cueing algorithm," IEEE transactions on cybernetics, vol. 49, no. 9, pp. 3471-3481, 2018.

[25] M. R. C. Qazani, H. Asadi, S. Khoo, and S. Nahavandi, "A Linear Time-Varying Model Predictive Control-Based Motion Cueing Algorithm for Hexapod Simulation-Based Motion Platform," IEEE Transactions on Systems, Man, and Cybernetic: Systems, 2019.

[26] M. R. C. Qazani, H. Asadi, and S. Nahavandi, "A Model Predictive Control-Based Motion Cueing Algorithm with Consideration of Joints' limitations for Hexapod Motion Platform," in 2019 IEEE International Conference on Systems, Man and Cybernetics (SMC), 2019, pp. 708713: IEEE.

[27] A. Mohammadi, S. Asadi, K. Nelson, and S. Nahavandi, "Future reference prediction in model predictive control based driving simulators," in Australasian conference on robotics and automation (ACRA2016), 2016.

[28] M. R. C. Qazani, H. Asadi, and S. Nahavandi, "A decoupled linear model predictive control-based motion cueing algorithm for simulation-based motion platform with limitted workspace," in IEEE International Conference on Systems, Man, and Cybernetics (SMC), Bari, Italy, 2019, pp. 1-6.

[29] P. R. MacNeilage, M. S. Banks, D. R. Berger, and H. H. Bülthoff, "A Bayesian model of the disambiguation of gravitoinertial force by visual cues," Experimental Brain Research, vol. 179, no. 2, pp. 263-290, 2007.

[30] H. Asadi, S. Mohamed, C. P. Lim, and S. Nahavandi, "A review on otolith models in human perception," Behavioural brain research, vol. 309, pp. 67-76, 2016.

[31] R. J. Telban and F. M. Cardullo, "Motion cueing algorithm development: Human-centered linear and nonlinear approaches," NASA TechReport CR-2005-213747, 2005.

[32] H. Asadi, S. Mohamed, C. P. Lim, S. Nahavandi, and E. Nalivaiko, "Semicircular canal modeling in human perception," Reviews in the Neurosciences, vol. 28, no. 5, pp. 537-549, 2017.

[33] B. Conrad and S. F. Schmidt, "A study of techniques for calculating motion drive signals for flight simulators," 1971.

[34] H. Asadi et al., "A Model Predictive Control-based Motion Cueing Algorithm using an optimized Nonlinear Scaling for Driving Simulators," in 2019 IEEE International Conference on Systems, Man and Cybernetics (SMC), 2019, pp. 1245-1250: IEEE.

[35] H. Asadi, C. P. Lim, A. Mohammadi, S. Mohamed, S. Nahavandi, and L. Shanmugam, "A genetic algorithm-based nonlinear scaling method for optimal motion cueing algorithm in driving simulator," Proceedings of the Institution of Mechanical Engineers, Part I: Journal of Systems and Control Engineering, vol. 232, no. 8, pp. 1025-1038, 2018.

[36] H. D. Kabir, A. Khosravi, S. Nahavandi, and A. Kavousi-Fard, "Partial Adversarial Training for Neural Network-Based Uncertainty Quantification," IEEE Transactions on Emerging Topics in Computational Intelligence, 2019.

[37] H. D. Kabir, A. Khosravi, M. A. Hosen, and S. Nahavandi, "Neural network-based uncertainty quantification: A survey of methodologies and applications," IEEE access, vol. 6, pp. 36218-36234, 2018.

[38] H. D. Kabir, A. Khosravi, M. A. Hosen, and S. Nahavandi, "Partial adversarial training for prediction interval," in 2018 International Joint Conference on Neural Networks (IJCNN), 2018, pp. 1-6: IEEE.

[39] H. M. D. Kabir and S. M. Salahuddin, "A frequency multiplier using three ambipolar graphene transistors," Microelectronics journal, vol. 70, pp. 12-15, 2017.

[40] H. D. Kabir, Z. Ahmed, R. Kariyadan, L. Zhang, and M. Chan, "Modeling of fringe current for semiconductor-extended organic TFTs," in 2016 IEEE International Conference on Electron Devices and Solid-State Circuits (EDSSC), 2016, pp. 177-180: IEEE.

[41] H. Kabir, A. Khosravi, A. Kavousi-Fard, S. Nahavandi, and D Srinivasan, "Optimal Uncertainty-guided Neural Network Training," arXiv preprint arXiv:1912.12761, 2019. 